\title{
Cyclic Product Theorems for Polygons (I) Constructions using Circles
}

\author{
G. C. Shephard \\ School of Mathematics, University of East Anglia, \\ Norwich NR4 7TJ, England, U.K. \\ G.C.Shephard@uea.ac.uk
}

\begin{abstract}
If a point $U_{i}$ is chosen on each edge of a plane $n$-gon $P$, then the product of the $n$ signed ratios in which the points $U_{i}$ divide the edges is called a cyclic product for $P$. The basic problem is to find geometrical constructions such that, for every $n$-gon $P$, the corresponding cyclic products either take constant values or satisfy simple relations. Many straight-line constructions are known. Here we describe some constructions which also involve circles.
\end{abstract}

\section{Introduction}

Let $P=\left[V_{0}, \ldots, V_{n-1}\right]$ be an $n$-gon in the Euclidean plane, and let $k$ be a positive integer $(k<n)$. Also suppose that, for $i=0, \ldots, n-1$, some geometric construction leads to a point $U_{i}$ on the edge or diagonal $V_{i} V_{i+k}$ of $P$. Then we shall use the term cyclic product (for $P$ ) to mean an expression of the form $\prod\left(\left\|V_{i} U_{i}\right\| /\left\|U_{i} V_{i+k}\right\|\right)$. Here and throughout, when considering $n$-gons, the subscripts are to be taken modulo $n$ and the product is from $i=0$ to $i=n-1$. The double modulus signs show that signed or directed lengths of the indicated segments are to be used. Thus, assuming the (collinear) points $V_{i}, U_{i}$, $V_{i+k}$ are distinct, $\left\|V_{i} U_{i}\right\| /\left\|U_{i} V_{i+k}\right\|$ is positive if and only if $U_{i}$ lies between $V_{i}$ and $V_{i+k}$.

Familiar examples of cyclic products which take a constant value are given by Menelaus' and Ceva's theorems [6, Chapter 8]. In Menelaus' theorem, $U_{i}$ is the point of intersection of an arbitrary fixed line with $V_{i} V_{i+1}$ and then $\prod\left(\left\|V_{i} U_{i}\right\| /\left\|U_{i} V_{i+1}\right\|\right)=$ $(-1)^{n}$; in Ceva's theorem, if $n=2 s+1$ is odd and the lines $V_{i} U_{i+s}$ are concurrent, then $\prod\left(\left\|V_{i} U_{i}\right\| /\left\|U_{i} V_{i+1}\right\|\right)=1$.

Dozens of other examples of cyclic products for $n$-gons are known (see [4], [5] and references given there). In all of these the points $U_{i}$ are determined by straight-line constructions (as with Menelaus' and Ceva's theorems). Here we shall be concerned with a situation in which the points $U_{i}$ are determined by constructions involving circles. Some of our results were conjectured or proved by Branko Grünbaum and appear in 
his (unpublished) lecture notes [3]. Details and acknowledgements appear in the final section of this paper.

Our cyclic products are what Howard Eves calls h-expressions [1, p. 247]. These are clearly affine invariants, but Eves proves the remarkable fact that they are also projective invariants. The simplest example of an h-expression is the cross-ratio of four points on a line.

In this paper we restrict ourselves to cases where the value of a cyclic product, or the product of two cyclic products, takes a fixed numerical value. Clearly, there will exist other relations involving more than two such products, but to search for these would make the investigation unwieldy. In fact, although we have made a serious effort to find all relations involving one or two cyclic products, it is almost certain that others exist, especially if one admits more and more complicated constructions.

To avoid repetition we shall now introduce some notation that is used throughout. The letter $P$ will always represent an $n$-gon with vertices $V_{0}, V_{1}, \ldots, V_{n-1}$. If $X, Y$ are distinct points, then $X Y$ is the line through $X$ and $Y,[X, Y]$ is the closed line segment with end points $X$ and $Y$, and $|X Y|$ will be used to mean the unsigned length of this segment so that $\pm|X Y|=\|X Y\|$. Similarly, for three non-collinear points $X, Y, Z$, we shall use $|X Y Z|$ and $\|X Y Z\|$ to mean the unsigned and signed area of the triangle $[X, Y, Z]$. We recall that the signed area of a simple polygon is positive if its vertices are named in a counterclockwise direction, and negative if named in a clockwise direction.

The polygons we consider are quite general: vertices may be collinear or coincide, edges may intersect at relative interior points or overlap, etc. We make only two requirements. The first is that no three consecutive vertices of a polygon $P$ are collinear, and the second depends on the construction under consideration, namely, that everything is well defined. Hence, in particular whenever a line is defined as the join of two points we shall suppose those points are distinct; when a point is defined as the intersection of two lines, then those two lines must not be parallel. When a circle is defined as passing through three points, then those points must not be collinear. Finally, in every cyclic product that occurs we shall suppose that none of the denominators vanish. It would, of course, be possible to specify, in each theorem, the exact restrictions that must hold, but as they are essentially obvious, to do so would be merely tedious and needlessly complicate the exposition without increasing its clarity.

The twelve theorems given here are divided into four types, but this is a matter of convenience only, and there is no doubt that other equally good classifications could be found.

We finish this Introduction with two remarks. The first of these enables us, in effect, to nearly double the number of theorems in the paper. If $V_{i}, V_{i+k}, U_{i}, W_{i}$ are four collinear points such that $\left\|V_{i} U_{i}\right\| /\left\|U_{i} V_{i+k}\right\|=-\left\|V_{i} W_{i}\right\| /\left\|W_{i} V_{i+k}\right\|$, then these four points are said to form a harmonic range, and $W_{i}$ is said to be the harmonic conjugate of $U_{i}$ with respect to $V_{i}$ and $V_{i+k}$, Clearly, $\prod\left(\left\|V_{i} U_{i}\right\| /\left\|U_{i} V_{i+k}\right\|\right)=(-1)^{n} \prod\left(\left\|V_{i} W_{i}\right\| /\left\|W_{i} V_{i+k}\right\|\right)$ and so each relation for cyclic products involving the points $U_{i}$ can be translated into another involving the points $W_{i}$. In some cases (such as the theorems of Section 2) these harmonic conjugates arise in a very natural way; in others (such as those in Section 1) they do not do so, and in such cases we make no further mention of cyclic product relations derived using harmonic conjugates in this manner.

The second remark is that many cyclic product relations involving one such product can be transformed into one involving two products by a process we call asymmetrisation. 
For example if a construction involving vertices $V_{i-r}, V_{i}, V_{i+r}$ of the polygon $P$ leads to a relation involving one cyclic product, then sometimes we can find a relation involving two cyclic products by using the same construction twice, once with $V_{i-r}, V_{i}, V_{i+s}$ and once with $V_{i-s}, V_{i}, V_{i+r}$. When this is possible we give a brief statement; the proofs are minor modifications of the symmetric case.

\section{Circle Constructions of the First Kind}

Each of the four theorems in this section use either a fixed circle, or one that is defined by three points. All the theorems depend on what is known as the power of a point with respect to a circle $Q$. We recall the definition. Let $Q$ be a given circle and $X_{0}$ be any given point. Let $m$ be a (variable) line through $X_{0}$ which intersects $Q$ in the points $Y$ and $Z$. Then the power $p\left(X_{0}, Q\right)$ of $X_{0}$ with respect to $Q$ is defined by $p\left(X_{0}, Q\right)=\left\|X_{0} Y\right\| \cdot\left\|X_{0} Z\right\|$. The most important property of the power is that its value depends only on the point $X_{0}$ and the circle $Q$, and not on the line $m$ used to define it. If $X_{0}$ lies inside the circle $Q$, then $p\left(X_{0}, Q\right)$ is negative. If $X_{0}$ lies outside $Q$, then $p\left(X_{0}, Q\right)$ is positive-it is the square of the length of a tangent from $X_{0}$ to $Q$. Notice that in this case $m$ must, of course, be chosen so as to intersect $Q$.

Theorem 1.1. Let $P$ be a given n-gon and $Q$ be a fixed circle. Suppose that, for each $i$, the side $V_{i} V_{i+1}$ of $P$ meets $Q$ in the points $U_{i}$ and $W_{i}$. Then

$$
\prod \frac{\left\|V_{i} U_{i}\right\|}{\left\|U_{i} V_{i+1}\right\|} \cdot \prod \frac{\left\|V_{i} W_{i}\right\|}{\left\|W_{i} V_{i+1}\right\|}=1
$$

Proof. See Fig. 1 for an example with $n=5$.

$$
\prod \frac{\left\|V_{i} U_{i}\right\|}{\left\|U_{i} V_{i+1}\right\|} \cdot \prod \frac{\left\|V_{i} W_{i}\right\|}{\left\|W_{i} V_{i+1}\right\|}=\prod \frac{\left\|V_{i} U_{i}\right\| \cdot\left\|V_{i} W_{i}\right\|}{\left\|U_{i} V_{i+1}\right\| \cdot\left\|W_{i} V_{i+1}\right\|}=\prod \frac{p\left(V_{i}, Q\right)}{p\left(V_{i+1}, Q\right)}=1 .
$$

The last equality follows since the terms in the numerator of the product are identical with those in the denominator, and so complete cancellation takes place.

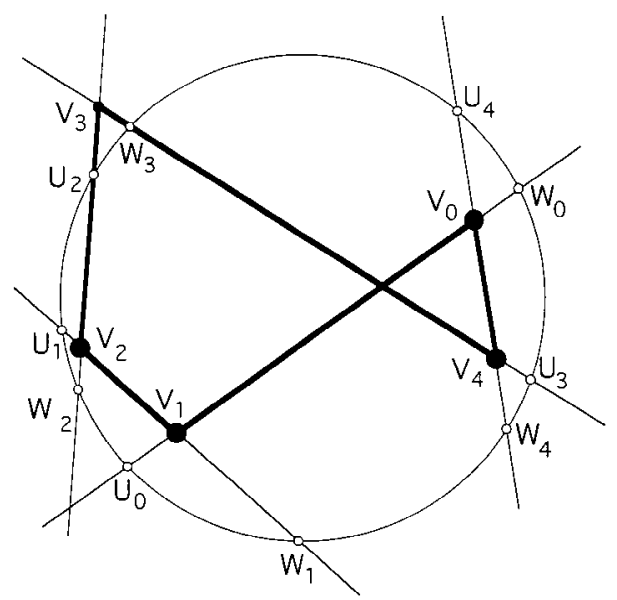

Fig. 1 
Note that, in the above, the points $U_{i}$ and $W_{i}$ can be interchanged without affecting the result.

Theorem 1.1 is a classical theorem in projective geometry due to Carnot, see p. 299 of [1], who stated it for triangles $(n=3)$ and $Q$ a proper conic. The latter is not a significant generalisation since by Eve's theorem, mentioned above, the cyclic products are projective invariants and so the statement of the theorem remains true if a projective transformation is applied to the figure. The $n$-gonal form of the theorem is also mentioned by Eves [1, Exercise 6.2-16]. It is included here since it clearly belongs to the same "family" of theorems as the remaining theorems of this section.

It would appear, at first sight, that we can generalise Theorem 1.1 by considering the diagonal $V_{i} V_{i+k}$ (where $k$ is a suitably chosen integer) instead of the side $V_{i} V_{i+1}$. However, this generalisation is illusory-if $k$ is prime to $n$ it is equivalent to applying the original theorem to the "stellated" polygon $\left[V_{0}, V_{k}, V_{2 k}, \ldots, V_{(n-1) k}\right]$. If $k$ is not prime to $n$ it is equivalent to applying the theorem to a suitable set of $h$ polygons, where $h=\operatorname{HCF}(k, n)(=\operatorname{GCD}(k, n))$. Similar remarks apply to all the other theorems in this section.

For the next three theorems we require the following lemma:

Lemma 1. Let the circle $Q$ have the equation

$$
f(x, y)=x^{2}+y^{2}-2 a x-2 b y+c=0
$$

(where $a^{2}+b^{2}>c$ and the coefficient of $x^{2}+y^{2}$ is equal to 1$)$. If $X_{0}$ is the point $\left(x_{0}, y_{0}\right)$, then $p\left(X_{0}, Q\right)=f\left(x_{0}, y_{0}\right)$.

Proof. See Fig. 2. If the radius of $Q$ is $r$ and the distance of $X_{0}$ from the centre $O$ of $Q$ is $s$, then, by considering the case where $m$ is tangent to $Q$ (when $X_{0}$ is outside the circle) or $m$ is perpendicular to $O X_{0}$ (when $X_{0}$ is inside the circle), we see immediately that $p\left(X_{0}, Q\right)=s^{2}-r^{2}$. However, as $O$ is the point $(a, b)$ and the radius of the circle is $\sqrt{ }\left(a^{2}+b^{2}-c\right)$, it follows that

$$
p\left(X_{0}, Q\right)=\left(x_{0}-a\right)^{2}+\left(y_{0}-b\right)^{2}-\left(a^{2}+b^{2}-c\right)=f\left(x_{0}, y_{0}\right)
$$

as required.

The lemma gives a simple method of calculating the power $p\left(X_{0}, Q\right)$ of the point $X_{0}\left(x_{0}, y_{0}\right)$ with respect to the circle $Q\left(X_{1}, X_{2}, X_{3}\right)$ through the three points $X_{1}\left(x_{1}, y_{1}\right)$, $X_{2}\left(x_{2}, y_{2}\right), X_{3}\left(x_{3}, y_{3}\right)$. The equation of $Q\left(X_{1}, X_{2}, X_{3}\right)$ is

$$
D\left(X, X_{1}, X_{2}, X_{3}\right)=\operatorname{det}\left|\begin{array}{cccc}
x^{2}+y^{2} & x & y & 1 \\
x_{1}^{2}+y_{1}^{2} & x_{1} & y_{1} & 1 \\
x_{2}^{2}+y_{2}^{2} & x_{2} & y_{2} & 1 \\
x_{3}^{2}+y_{3}^{2} & x_{3} & y_{3} & 1
\end{array}\right|=0 .
$$

In the expansion of the determinant, the coefficient of $x^{2}+y^{2}$ is

$$
\operatorname{det}\left|\begin{array}{lll}
x_{1} & y_{1} & 1 \\
x_{2} & y_{2} & 1 \\
x_{3} & y_{3} & 1
\end{array}\right| \quad \text { or } \quad 2\left\|X_{1} X_{2} X_{3}\right\|
$$




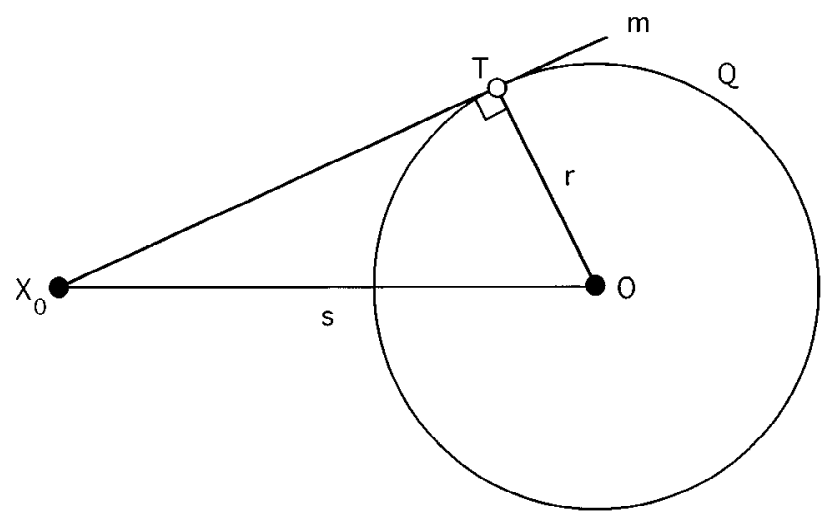

(a)

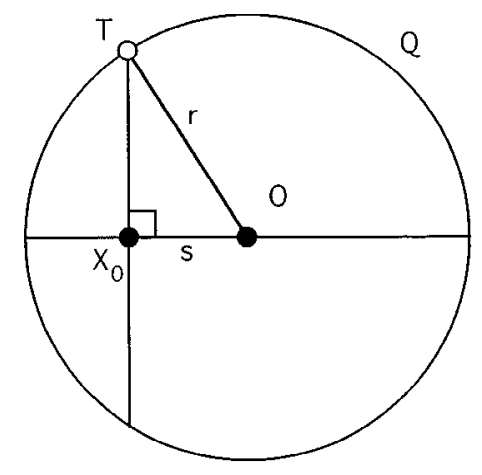

(b)

Fig. 2

Hence

$$
p\left(X_{0}, Q\left(X_{1}, X_{2}, X_{3}\right)\right)=\frac{D\left(X_{0}, X_{1}, X_{2}, X_{3}\right)}{2\left\|X_{1} X_{2} X_{3}\right\|} .
$$

This leads at once to an interesting identity which we have not seen in the literature, namely,

$$
\begin{aligned}
& p\left(X_{0}, Q\left(X_{1}, X_{2}, X_{3}\right)\right) \cdot\left\|X_{1} X_{2} X_{3}\right\| \\
& =p\left(X_{1}, Q\left(X_{2}, X_{3}, X_{0}\right)\right) \cdot\left\|X_{2} X_{3} X_{0}\right\| \\
& =p\left(X_{2}, Q\left(X_{3}, X_{0}, X_{1}\right)\right) \cdot\left\|X_{3} X_{0} X_{1}\right\| \\
& \quad=p\left(X_{3}, Q\left(X_{0}, X_{1}, X_{2}\right)\right) \cdot\left\|X_{0} X_{1} X_{2}\right\| .
\end{aligned}
$$




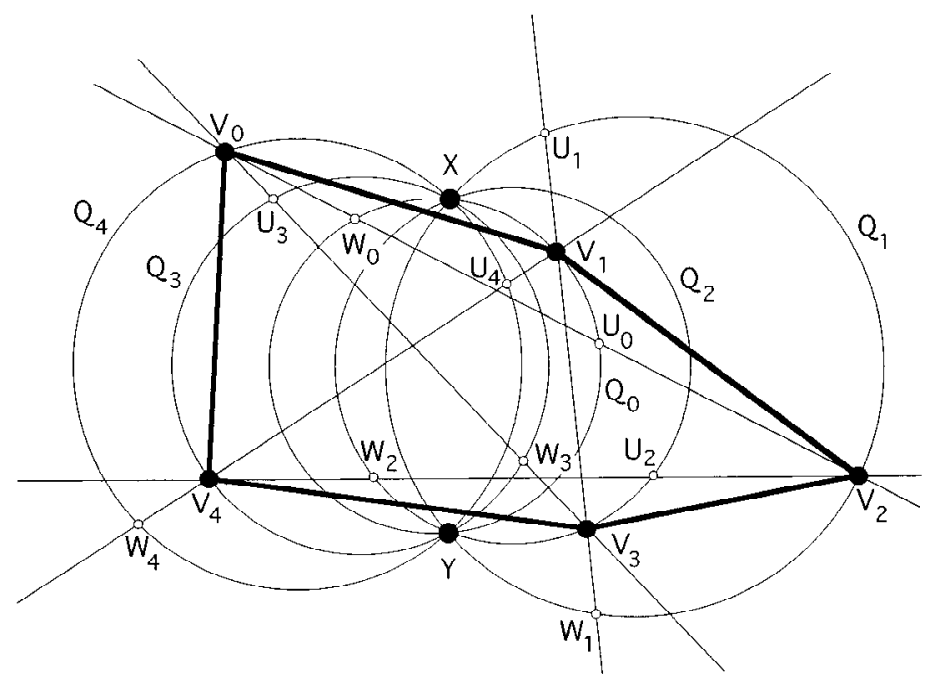

Fig. 3

Theorem 1.2. Let $P$ be a given $n$-gon, and $X, Y$ be two fixed points. For each $i=$ $0, \ldots, n-1$, define $Q_{i}$ as the (unique) circle through $V_{i+1}, X$ and $Y$, and suppose that the diagonal $V_{i} V_{i+2}$ of $P$ meets $Q_{i}$ in the points $U_{i}$ and $W_{i}$. Then

$$
\prod \frac{\left\|V_{i} U_{i}\right\|}{\left\|U_{i} V_{i+2}\right\|} \cdot \prod \frac{\left\|V_{i} W_{i}\right\|}{\left\|W_{i} V_{i+2}\right\|}=(-1)^{n}
$$

Proof. See Fig. 3 for an example with $n=5$.

$$
\frac{\left\|V_{i} U_{i}\right\|}{\left\|U_{i} V_{i+2}\right\|} \cdot \frac{\left\|V_{i} W_{i}\right\|}{\left\|W_{i} V_{i+2}\right\|}=\frac{p\left(V_{i}, Q_{i}\right)}{p\left(V_{i+2}, Q_{i}\right)}=\frac{D\left(V_{i}, V_{i+1}, X, Y\right) \cdot\left\|V_{i+1} X Y\right\|}{D\left(V_{i+2}, V_{i+1}, X, Y\right) \cdot\left\|V_{i+1} X Y\right\|}
$$

and so

$$
\prod \frac{\left\|V_{i} U_{i}\right\|}{\left\|U_{i} V_{i+2}\right\|} \cdot \prod \frac{\left\|V_{i} W_{i}\right\|}{\left\|W_{i} V_{i+2}\right\|}=(-1)^{n} \prod \frac{D\left(V_{i}, V_{i+1}, X, Y\right)}{D\left(V_{i+1}, V_{i+2}, X, Y\right)}=(-1)^{n} .
$$

The last equality follows since all the terms in the product cancel and so the theorem is established.

Theorem 1.3. Let $P$ be a given $n$-gon $(n \geq 4)$ and let $X$ be a fixed point. For each $i=0, \ldots, n-1$ let $Q_{i}$ be the (unique) circle through $X, V_{i+1}, V_{i+2}$, and let $U_{i}, W_{i}$ be the two points in which the diagonal $V_{i} V_{i+3}$ of $P$ meets $Q_{i}$. Then

$$
\prod \frac{\left\|V_{i} U_{i}\right\|}{\left\|U_{i} V_{i+3}\right\|} \cdot \prod \frac{\left\|V_{i} W_{i}\right\|}{\left\|W_{i} V_{i+3}\right\|}=1 \text {. }
$$


Proof. The proof is similar to that of Theorem 1.2 using

$$
\frac{\left\|V_{i} U_{i}\right\|}{\left\|U_{i} V_{i+3}\right\|} \cdot \frac{\left\|V_{i} W_{i}\right\|}{\left\|W_{i} V_{i+3}\right\|}=\frac{p\left(V_{i}, Q_{i}\right)}{p\left(V_{i+3}, Q_{i}\right)}=\frac{D\left(V_{i}, V_{i+1}, V_{i+2}, X\right) \cdot\left\|V_{i+1} V_{i+2} X\right\|}{D\left(V_{i+3}, V_{i+1}, V_{i+2}, X\right) \cdot\left\|V_{i+1} V_{i+2} X\right\|}
$$

instead of (1).

Theorem 1.4. Let $P$ be a given $n$-gon $(n \geq 5)$ and for each $i=0, \ldots, n-1$ let $Q_{i}$ be the (unique) circle through the three vertices $V_{i+1}, V_{i+2}, V_{i+3}$ of $P$. Suppose the diagonal $V_{i} V_{i+4}$ of $P$ meets $Q_{i}$ in $U_{i}$ and $W_{i}$. Then

$$
\prod \frac{\left\|V_{i} U_{i}\right\|}{\left\|U_{i} V_{i+4}\right\|} \cdot \prod \frac{\left\|V_{i} W_{i}\right\|}{\left\|W_{i} V_{i+4}\right\|}=(-1)^{n} .
$$

Proof. The proof is similar to that of Theorem 1.2 using

$$
\frac{\left\|V_{i} U_{i}\right\|}{\left\|U_{i} V_{i+4}\right\|} \cdot \frac{\left\|V_{i} W_{i}\right\|}{\left\|W_{i} V_{i+4}\right\|}=\frac{p\left(V_{i}, Q_{i}\right)}{p\left(V_{i+4}, Q_{i}\right)}=\frac{D\left(V_{i}, V_{i+1}, V_{i+2}, V_{i+3}\right) \cdot\left\|V_{i+1} V_{i+2} V_{i+3}\right\|}{D\left(V_{i+4}, V_{i+1}, V_{i+2}, V_{i+3}\right) \cdot\left\|V_{i+1} V_{i+2} V_{i+3}\right\|}
$$

instead of (1).

If, in each of the above theorems, the points $U_{i}$ and $W_{i}$ are not defined because the edge or diagonal $V_{i} V_{i+k}$ does not meet the circle $Q_{i}$, then instead of $\left(\left\|V_{i} U_{i}\right\| \cdot\left\|V_{i} W_{i}\right\|\right) /\left(\left\|U_{i} V_{i+k}\right\| \cdot\left\|W_{i} V_{i+k}\right\|\right)$ we may write $t_{i}^{2} / t_{i+k}^{2}$ where $t_{i}$ and $t_{i+k}$ are the lengths of the tangents from $V_{i}$ and $V_{i+k}$ to $Q_{i}$. This follows since both expressions are equal to $p\left(V_{i}, Q_{i}\right) / p\left(V_{i+k}, Q_{i}\right)$. However, if this is done, the theorems become assertions about powers of vertices with respect to the circles instead of statements about cyclic products.

\section{Circle Constructions of the Second Kind}

The constructions here, like those of the previous section, involve circles drawn through three points. Here we also use tangents to the circles at one of the points.

Lemma 2. If $Q$ is the circumcircle of the triangle $[A, B, C]$ and the tangent to $Q$ at $A$ meets the line $B C$ in $D$, then

$$
\frac{\|B D\|}{\|D C\|}=-\frac{|A B|^{2}}{|A C|^{2}}
$$

Proof. See Fig. 4. By elementary geometry the triangles $[B, A, D]$ and $[A, C, D]$ are similar, so $|B D| /|A D|=|A B| /|A C|$ and $|A D| /|D C|=|A B| /|A C|$. Multiplying these equations together we obtain $|B D| /|D C|=|B A|^{2} /|A C|^{2}$, and then the statement of the lemma follows from the definition of directed lengths.

Theorem 2.1. Let $P$ be a given n-gon and $X$ be a fixed point. For each $i=0, \ldots, n-1$ let $Q_{i}$ be the (unique) circle through the three points $V_{i}, V_{i+1}, X$. Let the tangent to $Q_{i}$ 


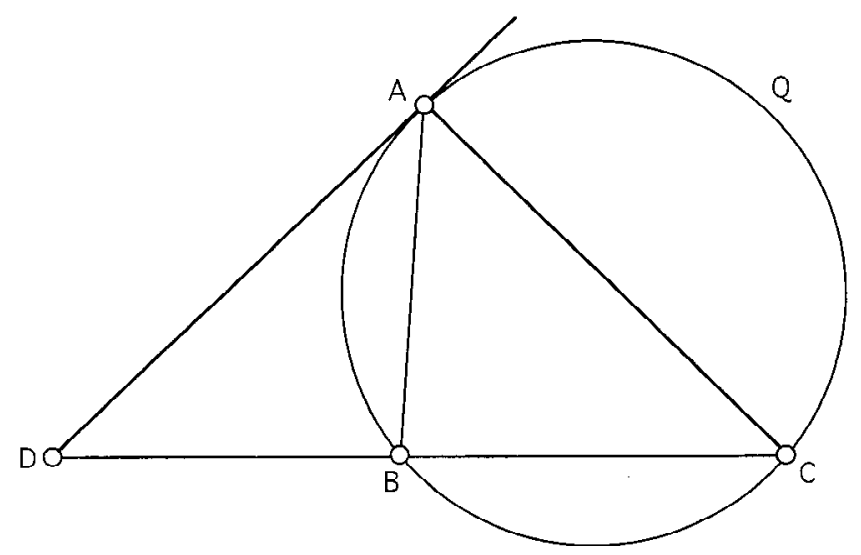

Fig. 4

at $X$ meet the side $V_{i} V_{i+1}$ of $P$ in $U_{i}$. Then

$$
\prod \frac{\left\|V_{i} U_{i}\right\|}{\left\|U_{i} V_{i+1}\right\|}=(-1)^{n}
$$

In Fig. 5(a) we show the construction of the point $U_{i}$, and in Fig. 5(b) the construction of all the points $U_{i}(i=0,1, \ldots, 4)$ for a pentagon. Many of the subsequent diagrams will follow the same arrangement. For each theorem we show the basic construction, and, in some cases, the complete construction for a pentagon. Where the complete construction is not shown this is because, even when $n$ is as small as 5, the resulting diagram is so complicated as to be unintelligible.

Proof. By Lemma 2, with $X, V_{i}, V_{i+1}$ written in place of $A, B, C$, respectively, we see that $D=U_{i}$ and hence

$$
\frac{\left\|V_{i} U_{i}\right\|}{\left\|U_{i} V_{i+1}\right\|}=-\frac{\left|X V_{i}\right|^{2}}{\left|X V_{i+1}\right|^{2}}
$$

Thus,

$$
\prod \frac{\left\|V_{i} U_{i}\right\|}{\left\|U_{i} V_{i+1}\right\|}=(-1)^{n} \prod \frac{\left|X V_{i}\right|^{2}}{\left|X V_{i+1}\right|^{2}}=(-1)^{n} .
$$

In the last product complete cancellation occurs and so the theorem is proved.

If, with the above notation, $Y_{i}$ is the point of contact of the second tangent from $U_{i}$ to $Q_{i}$ and the line $X Y_{i}$ meets the side $V_{i} V_{i+1}$ of $P$ in $W_{i}$, then $W_{i}$ is the harmonic conjugate of $U_{i}$ with respect to $V_{i}, V_{i+1}$ and so, by the remark in the Introduction, $\prod\left(\left\|V_{i} W_{i}\right\| /\left\|W_{i} V_{i+1}\right\|\right)=1$.

Theorem 2.2. Let $P$ be a given $n$-gon and for $i=0, \ldots, n-1$ let $Q_{i}$ be the (unique) circle through the three points $V_{i}, V_{i+1}, V_{i+2}$. Let the tangent to $Q_{i}$ at $V_{i+1}$ meet the line 


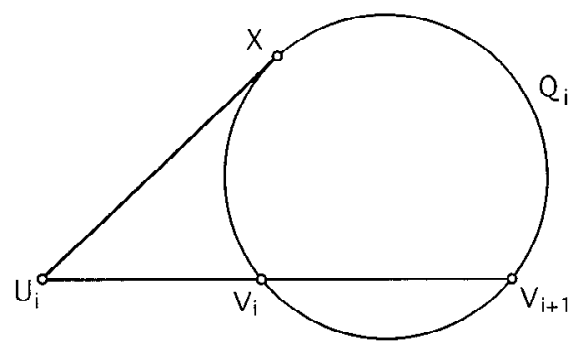

(a)

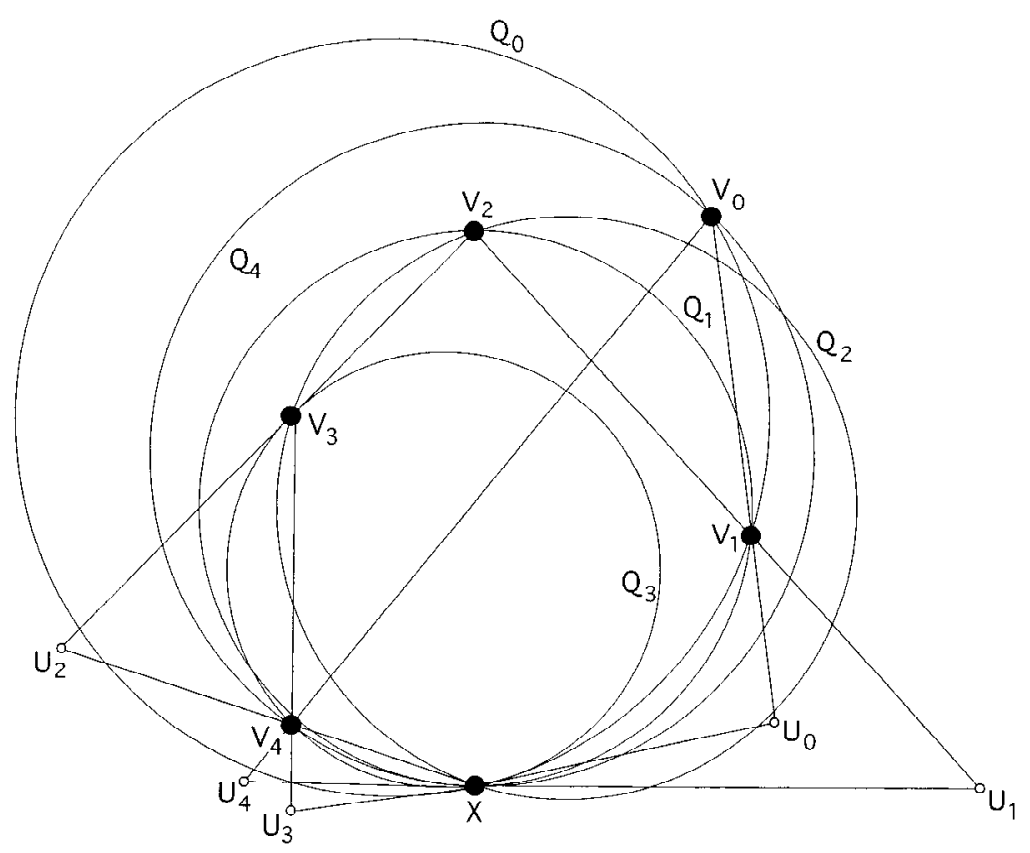

(b)

Fig. 5

$V_{i} V_{i+2}$ of $P$ in $U_{i}$. Then

$$
\prod \frac{\left\|V_{i} U_{i}\right\|}{\left\|U_{i} V_{i+2}\right\|}=(-1)^{n}
$$

Proof. The proof is similar to that of Theorem 2.1. Here we write $V_{i}, V_{i+1}, V_{i+2}$ in place of $B, A$ and $C$ in Lemma 2 , so that $D=U_{i}$, and in place of (2) we have

$$
\frac{\left\|V_{i} U_{i}\right\|}{\left\|U_{i} V_{i+2}\right\|}=-\frac{\left|V_{i+1} V_{i}\right|^{2}}{\left|V_{i+1} V_{i+2}\right|^{2}} .
$$

Taking the product from $i=0$ to $i=n-1$ yields the statement of the theorem. 
In the case $n=3$ of Theorem 2.2, which is well known, the points $U_{1}, U_{2}, U_{3}$ are collinear by the converse of Menelaus' theorem.

If, with the above notation, $Y_{i}$ is the point of contact of the second tangent from $U_{i}$ to $Q_{i}$ and the line $V_{i+1} Y_{i}$ meets the diagonal $V_{i} V_{i+2}$ of $P$ in $W_{i}$, then $W_{i}$ is the harmonic conjugate of $U_{i}$ with respect to $V_{i}, V_{i+2}$ and so, by the remark in the Introduction, $\prod\left(\left\|V_{i} W_{i}\right\| /\left\|W_{i} V_{i+2}\right\|\right)=1$.

Theorem 2.3. Let $P$ be an $n$-gon and $X$ be a fixed point. For each $i=0, \ldots, n-1$ let $Q_{i}$ be the (unique) circle through the points $V_{i}, V_{i+1}, X$. Let the tangent to $Q_{i}$ at $V_{i}$ meet the line $X V_{i+1}$ in $U_{i+1}$ and the tangent to $Q_{i}$ at $V_{i+1}$ meet the line $X V_{i}$ at $W_{i}$. Then

$$
\prod \frac{\left\|V_{i+1} U_{i+1}\right\|}{\left\|U_{i+1} X\right\|} \cdot \prod \frac{\left\|X W_{i}\right\|}{\left\|W_{i} V_{i}\right\|}=1 .
$$

Proof. See Fig. 6 for an example with $n=5$. The proof is similar to that of Theorem 2.1 except that we apply Lemma 2 twice, firstly with $V_{i}, V_{i+1}, X$ in place of $A, B, C$ of Lemma 2 (so that $D=U_{i+1}$ ) and secondly with $V_{i+1}, V_{i}, X$ in place of $A, B, C$ (so $\left.D=W_{i}\right)$. We obtain, in place of (2),

$$
\frac{\left\|V_{i+1} U_{i+1}\right\|}{\left\|U_{i+1} X\right\|}=-\frac{\left|V_{i} V_{i+1}\right|^{2}}{\left|V_{i} X\right|^{2}}
$$

and

$$
\frac{\left\|V_{i} W_{i}\right\|}{\left\|W_{i} X\right\|}=-\frac{\left|V_{i+1} V_{i}\right|^{2}}{\left|V_{i+1} X\right|^{2}} .
$$

Take the quotient of these, and then the product from $i=0$ to $i=n-1$ yields the statement in the theorem.

Theorem 2.2 has an asymmetric form:

Theorem 2.4. Let $P$ be an $n$-gon and $s$, $t$ be positive integers, $s<n / 2, t<n / 2, s \neq t$. For each $i=0, \ldots, n-1$ let $Q_{i}$ be the (unique) circle through the points $V_{i-s}, V_{i}, V_{i+t}$ and $R_{i}$ be the (unique) circle through $V_{i-t}, V_{i}, V_{i+s}$. Let the tangent to $Q_{i}$ at $V_{i}$ meet the line $V_{i-s} V_{i+t}$ in $U_{i}$ and the tangent to $R_{i}$ at $V_{i}$ meet the line $V_{i-t} V_{i+s}$ at $W_{i}$. Then

$$
\prod \frac{\left\|V_{i-s} U_{i}\right\|}{\left\|U_{i} V_{i+t}\right\|} \cdot \prod \frac{\left\|V_{i-t} W_{i}\right\|}{\left\|W_{i} V_{i+s}\right\|}=1 .
$$

Proof. See Fig. 7 for the construction of the points $U_{i}$ and $W_{i}$. The proof is similar to that of Theorem 2.1 except that we apply Lemma 2 twice, firstly with $V_{i}, V_{i-s}, V_{i+t}$ in place of $A, B, C$ (so that $D=U_{i}$ ) and secondly with $V_{i}, V_{i-t}, V_{i+s}$ in place of $A, B, C$, respectively (so that $D=W_{i}$ ). Taking the product of the two equations so obtained and then the product from $i=0$ to $i=n-1$ yields the statement in the theorem. 


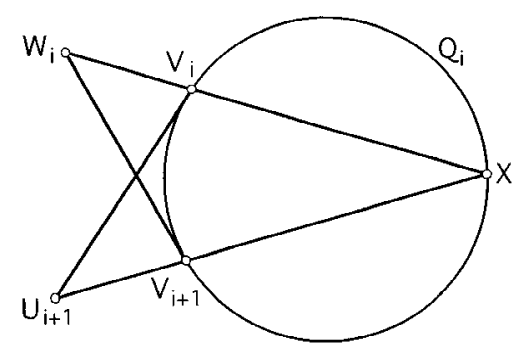

(a)

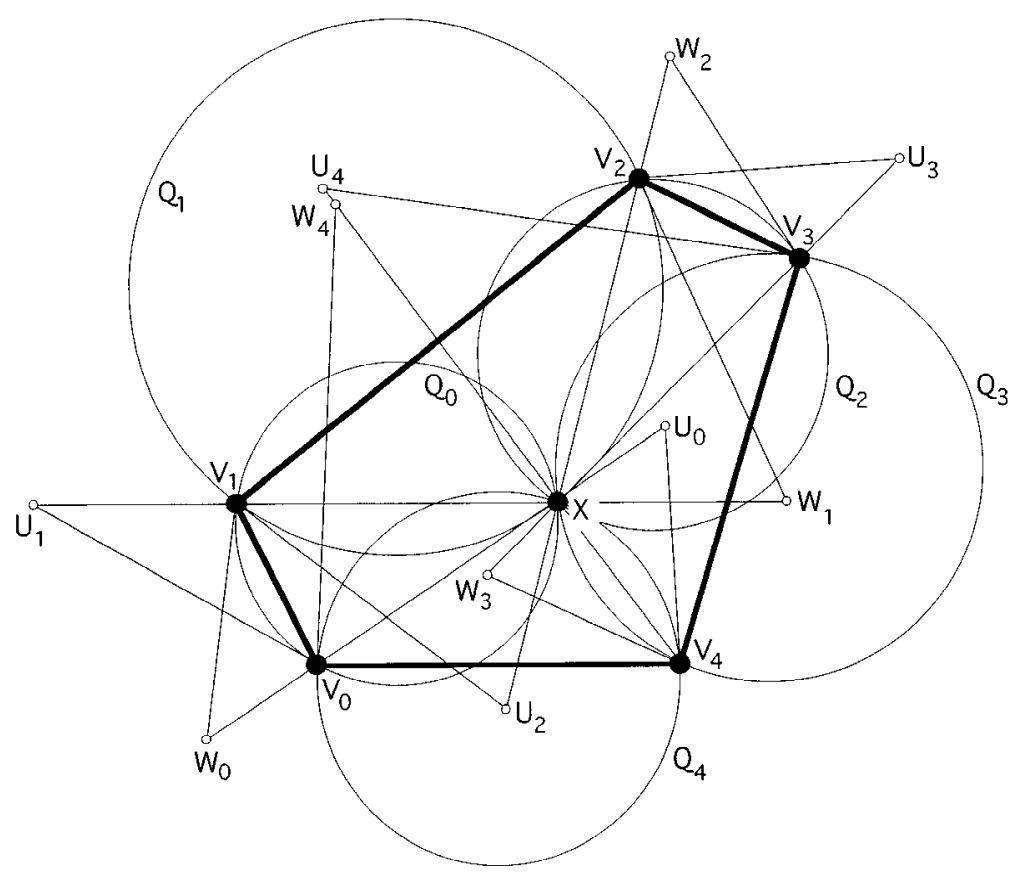

(b)

Fig. 6

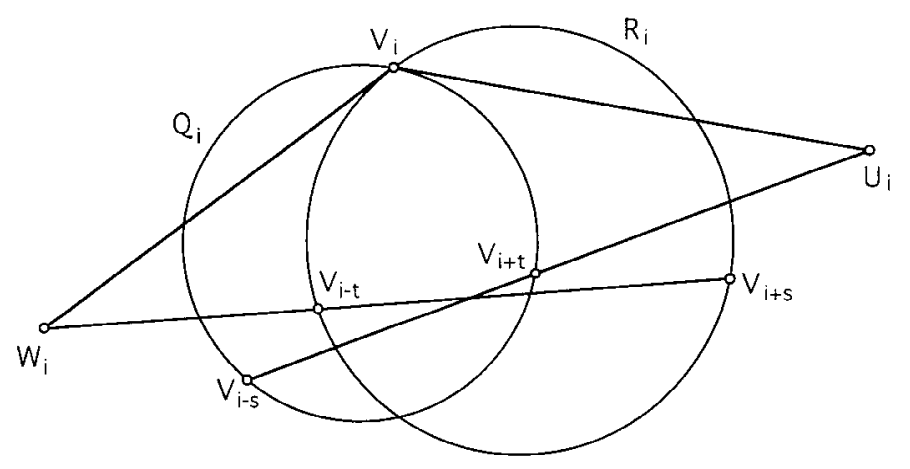

Fig. 7 


\section{Circle Constructions of the Third Kind}

Each of the two constructions described in this section involve circles which touch an edge of the polygon at one of the two vertices on that edge and satisfy one extra condition. More precisely, they touch the edge of a polygon $P$ at one of its vertices and either pass through another vertex or touch another edge of $P$.

Theorem 3.1. Let $P$ be a given $n$-gon $(n \geq 4)$ and for each $i=0, \ldots, n-1$ let $Q_{i}$ be the (unique) circle which touches the side $\left[V_{i}, V_{i+1}\right]$ of $P$ at the vertex $V_{i}$ and passes through $V_{i+2}$. Also, let $R_{i}$ be the (unique) circle which touches the same side $\left[V_{i}, V_{i+1}\right]$ at the vertex $V_{i+1}$ and passes through $V_{i-1}$. Let the second intersection of $Q_{i}$ with $V_{i+1} V_{i+2}$ be denoted by $U_{i}$ and the second intersection of $R_{i}$ with $V_{i} V_{i-1}$ be denoted by $W_{i}$. Then

$$
\prod \frac{\left\|V_{i+1} U_{i}\right\|}{\left\|U_{i} V_{i+2}\right\|} \cdot \prod \frac{\left\|V_{i-1} W_{i}\right\|}{\left\|W_{i} V_{i}\right\|}=(-1)^{n} .
$$

Proof. See Fig. 8. Assume that the positive direction on each edge $\left[V_{i}, V_{i+1}\right]$ is $\vec{V}_{i} V_{i+1}$ and for each $i=0, \ldots, n-1$ write $k_{i}=\left\|V_{i} V_{i+1}\right\|>0$. Then by properties of the power $p\left(V_{i+1}, Q_{i}\right)$ we see $\left\|V_{i+1} U_{i}\right\| \cdot\left\|V_{i+1} V_{i+2}\right\|=\left|V_{i+1} V_{i}\right|^{2}=k_{i}^{2}$ so that $\left\|V_{i+1} U_{i}\right\|=$ $k_{i}^{2} / k_{i+1}$. But,

$$
\left\|U_{i} V_{i+2}\right\|=\left\|U_{i} V_{i+1}\right\|+\left\|V_{i+1} V_{i+2}\right\|=-\frac{k_{1}^{2}}{k_{i+1}}+k_{i+1}=\frac{k_{i+1}^{2}-k_{i}^{2}}{k_{i+1}},
$$

and so $\left\|V_{i+1} U_{i}\right\| /\left\|U_{i} V_{i+2}\right\|=k_{i}^{2} /\left(k_{i+1}^{2}-k_{i}^{2}\right)$. In a similar way, $\left\|V_{i} W_{i}\right\| /\left\|W_{i} V_{i-1}\right\|=$ $k_{i}^{2} /\left(k_{i-1}^{2}-k_{i}^{2}\right)$, and therefore,

$$
\prod \frac{\left\|V_{i+1} U_{i}\right\|}{\left\|U_{i} V_{i+2}\right\|} \cdot \prod \frac{\left\|V_{i-1} W_{i}\right\|}{\left\|W_{i} V_{i}\right\|}=\prod \frac{k_{i-1}^{2}-k_{i}^{2}}{k_{i+1}^{2}-k_{i}^{2}}=(-1)^{n} \prod \frac{k_{i-1}^{2}-k_{i}^{2}}{k_{i}^{2}-k_{i+1}^{2}}=(-1)^{n}
$$

since all the terms in the last product cancel.

Theorem 3.2. Let $P$ be a given $n$-gon $(n \geq 4)$ and for each $i=0, \ldots, n-1$ let $Q_{i}$ be the (unique) circle which touches the side $V_{i}, V_{i+1}$ of $P$ at the vertex $V_{i}$ and also touches the side $V_{i+1} V_{i+2}$ (on the same side of $V_{i} V_{i+1}$ as $V_{i+2}$ ) at the point $U_{i}$. Let $R_{i}$ be the (unique) circle which touches the same side $V_{i}, V_{i+1}$ at the vertex $V_{i+1}$ and also touches

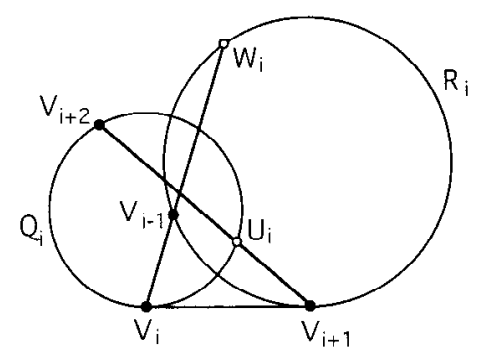

Fig. 8 
the side $V_{i} V_{i-1}$ (on the same side of $V_{i} V_{i+1}$ as $V_{i-1}$ ) at the point $W_{i}$. Then

$$
\prod \frac{\left\|V_{i+1} U_{i}\right\|}{\left\|U_{i} V_{i+2}\right\|} \cdot \prod \frac{\left\|V_{i-1} W_{i}\right\|}{\left\|W_{i} V_{i}\right\|}=(-1)^{n} .
$$

Proof. See Fig. 9 for an example with $n=5$. Define $k_{i}$ as in the proof of Theorem 3.1. Since tangents from an external point to a circle are equal, and, by the definition of $U_{i}$, $\left\|V_{i+1} U_{i}\right\|>0$, so $\left\|V_{i+1} U_{i}\right\|=k_{i}$. Also $\left\|V_{i+1} U_{i}\right\|+\left\|U_{i} V_{i+2}\right\|=\left\|V_{i+1} V_{i+2}\right\|=k_{i+1}$. Hence $\left\|U_{i} V_{i+2}\right\|=k_{i+1}-k_{i}$, and we obtain $\left\|V_{i+1} U_{i}\right\| /\left\|U_{i} V_{i+2}\right\|=k_{i} /\left(k_{i+1}-k_{i}\right)$. In a similar way, $\left\|V_{i-1} W_{i}\right\| /\left\|W_{i} V_{i}\right\|=\left(k_{i-1}-k_{i}\right) / k_{i}$, and so

$$
\prod \frac{\left\|V_{i+1} U_{i}\right\|}{\left\|U_{i} V_{i+2}\right\|} \cdot \prod \frac{\left\|V_{i-1} W_{i}\right\|}{\left\|W_{i} V_{i}\right\|}=\prod\left(-\frac{k_{i-1}-k_{i}}{k_{i}-k_{i+1}}\right)=(-1)^{n}
$$

since all the terms in the last product cancel.

\section{Circle Constructions of the Fourth Kind}

In Sections 1 and 2 we considered constructions in which the circles were determined by the condition that they pass through three points (fixed points or vertices of the given polygon $P$ ). We now consider constructions in which the circles are determined by the condition that they touch three lines (either fixed lines or sides of the polygon $P$ ). In this case the situation is more complicated; whereas there is a unique circle through three given points, there are, in general, four circles which touch three given lines (namely, the incircle and three excircles of the triangle formed by the three lines). This has the important consequence that we need to specify, in each of the theorems, exactly which circles are to be chosen. As we shall see there are many ways to do this, and as a result, each construction leads to many cyclic product relations.

Theorem 4.1. Let $m$ be a fixed line and $P$ be a given n-gon. For each $i=0,1, \ldots, n-1$ let $F_{i}$ be the point in which the side $V_{i-1} V_{i}$ of $P$ meets $m$. Let $Q_{i}$ be a circle, centre $O_{i}$, such that

(i) $Q_{i}$ touches the sides $V_{i-1} V_{i}, V_{i} V_{i+1}$ of $P$ and also the line $m$,

(ii) the points $O_{i}, F_{i+1}, O_{i+1}$ are collinear, and

(iii) $Q_{i}$ touches the line $m$ at the point $U_{i}$,

then

$$
\prod \frac{\left\|F_{i} U_{i}\right\|}{\left\|U_{i} F_{i+1}\right\|}=(-1)^{n}
$$

Proof. See Fig. 10 for an example with $n=5$. The points $O_{i-1}, F_{i}, O_{i}$ lie on one of the two bisectors of the angle between $m$ and the line $V_{i-1} V_{i}$ at $F_{i}$ (because both the circles $Q_{i-1}$ and $Q_{i}$ touch the lines $m$ and $V_{i-1} V_{i}$ ). There are two such bisectors at $F_{i}$ and we may choose either. Temporarily call these a-bisectors and b-bisectors (see Fig. 11). The 


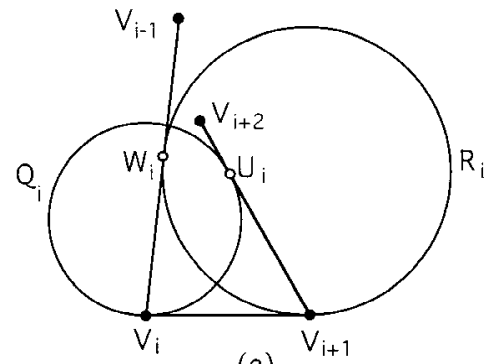

(a)

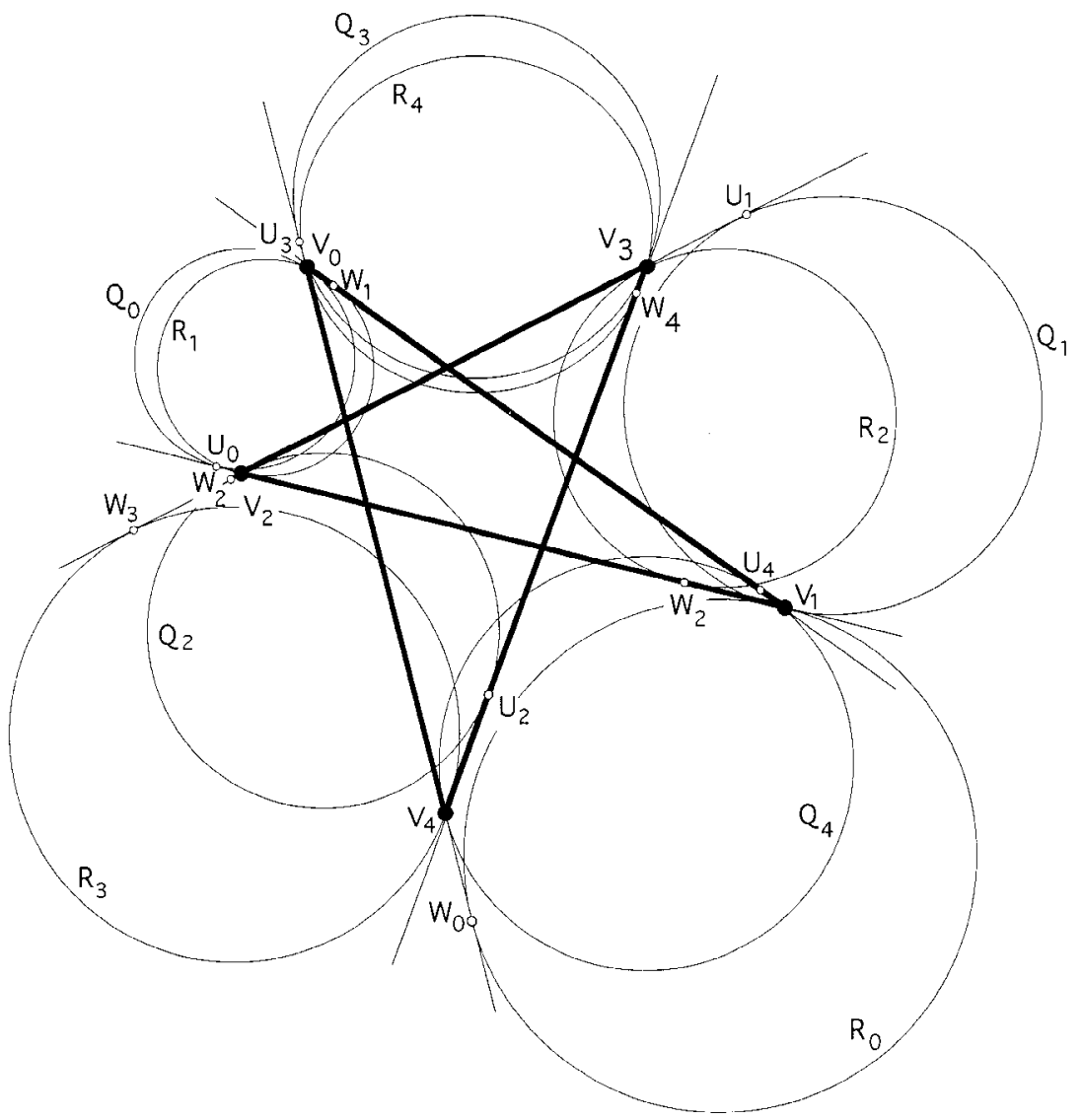

(b)

Fig. 9 


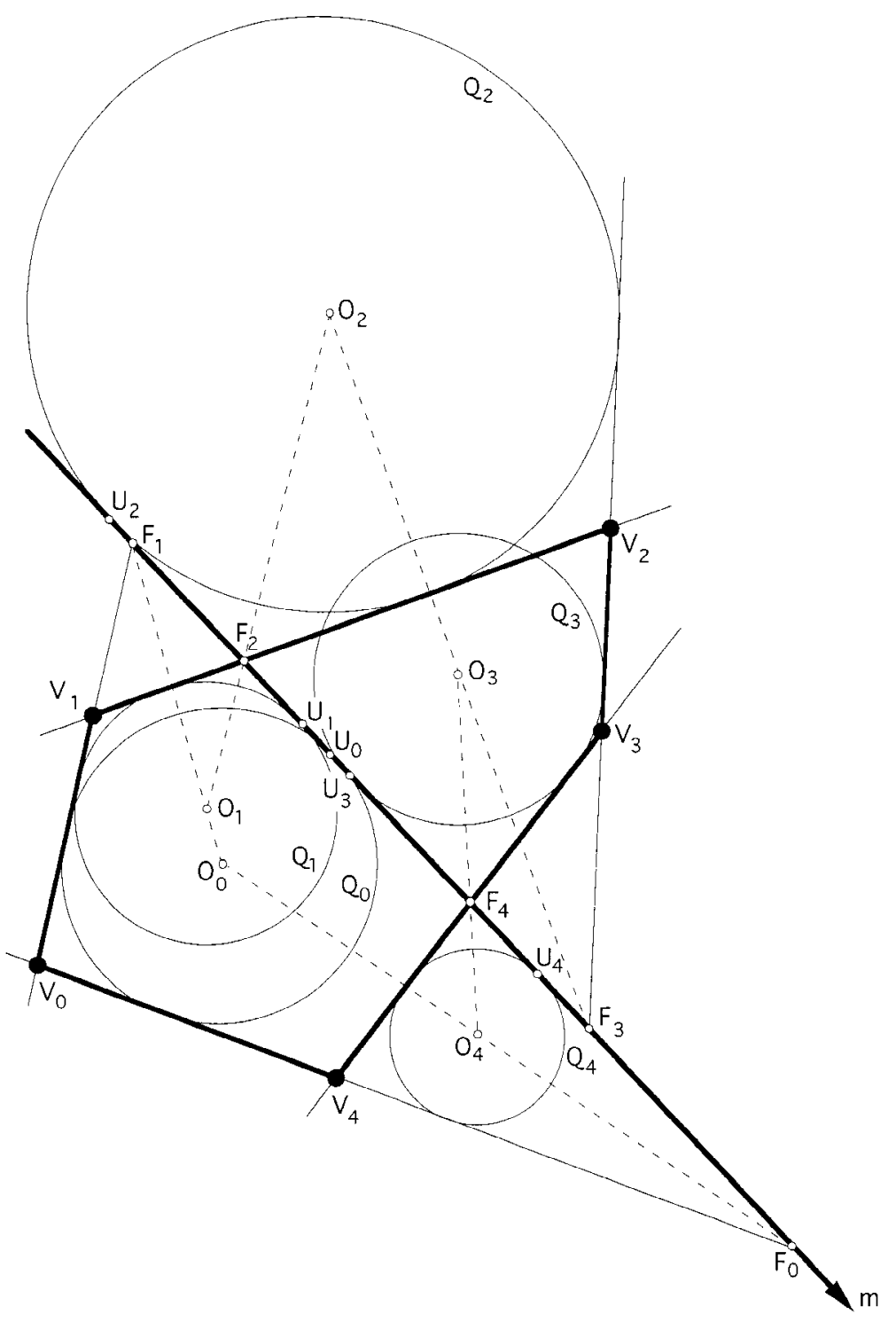

Fig. 10

two kinds are distinguished by the fact that if one moves in a counterclockwise direction about $F_{i}$, starting from the positive direction of $m$, then one meets the $a$-bisector before the line $V_{i-1} V_{i}$ but the $b$-bisector after this line. Define

$$
e_{i}= \begin{cases}1 & \text { if we choose the } a \text {-bisector at } F_{i} \\ -1 & \text { if we choose the } b \text {-bisector at } F_{i}\end{cases}
$$

As $O_{i}$, the centre of $Q_{i}$, lies on the bisectors at both $F_{i}$ and $F_{i+1}$ there are four possibilities 


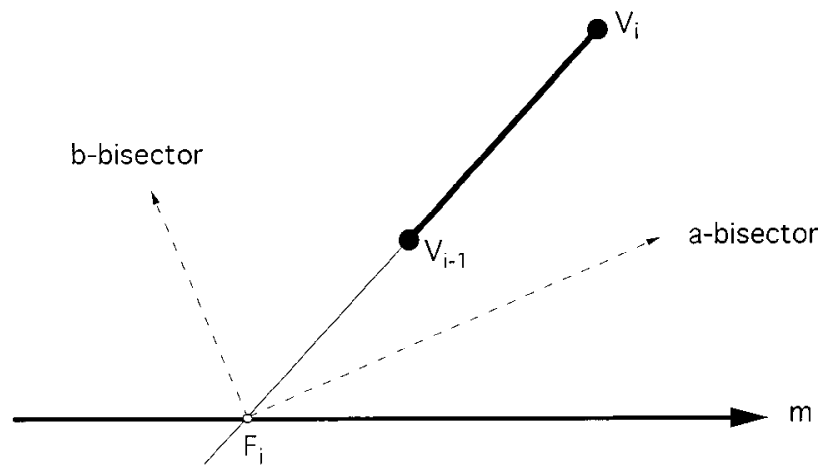

Fig. 11

according to which bisectors are chosen. These are shown in Fig. 12. With angles $\theta_{i}$ and $\theta_{i+1}$ as marked, and $r_{i}>0$ as the radius of $Q_{i}$, in every case

$$
\frac{\left\|F_{i} U_{i}\right\|}{\left\|U_{i} F_{i+1}\right\|}=-\frac{e_{i} e_{i+1} r_{i} \cot \theta_{i}}{r_{i} \cot \theta_{i+1}}
$$

and so

$$
\prod \frac{\left\|F_{i} U_{i}\right\|}{\left\|U_{i} F_{i+1}\right\|}=(-1)^{n} \prod e_{i} e_{i+1} \prod \frac{\cot \theta_{i}}{\cot \theta_{i+1}}=(-1)^{n}
$$

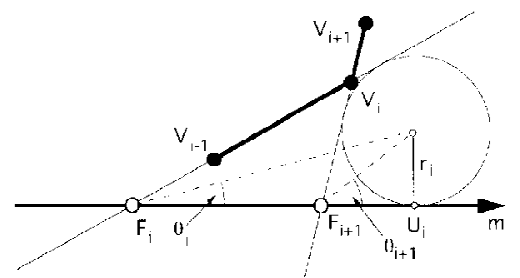

(a)

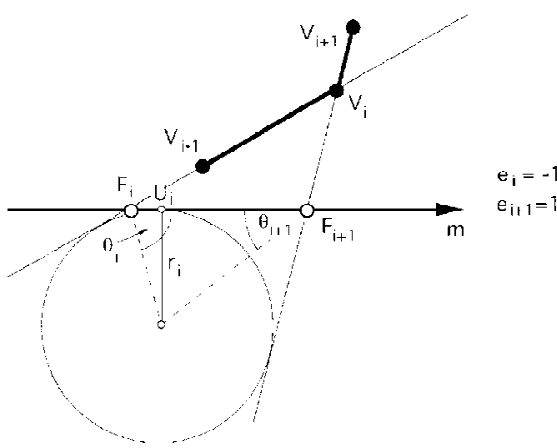

(c)

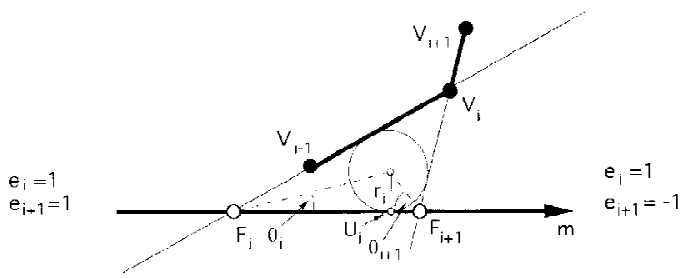

(b)

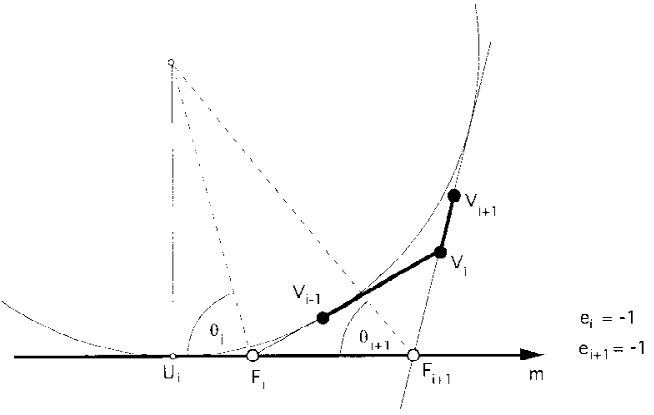

(d)

Fig. 12 
There are $2^{n}$ possible sets of bisectors that may be chosen at the points $F_{i}$. Each such set determines a sequence of circles $Q_{i}$ that satisfy the conditions of the theorem. We thus arrive at $2^{n}$ sets of points $U_{i}$, each of which leads to a cyclic product relation. Hence, in general, (3) represents $2^{n}$ such relations.

Theorem 4.2. Let $P$ be a given $n$-gon, and for each $i=0,1, \ldots, n-1$ let $Q_{i}$ be a circle, centre $O_{i}$, such that

(i) $Q_{i}$ touches the sides $V_{i-1} V_{i}, V_{i} V_{i+1}, V_{i+1} V_{i+2}$ of $P$,

(ii) the points $O_{i}, V_{i+1}, O_{i+1}$ are collinear, and

(iii) $Q_{i}$ touches the side $V_{i} V_{i+1}$ of $P$ at the point $U_{i}$.

Then

$$
\prod \frac{\left\|V_{i} U_{i}\right\|}{\left\|U_{i} V_{i+1}\right\|}=1
$$

Proof. See Fig. 13 for examples with $n=5$. In Fig. 13(a) the polygon is convex, and in Fig. 13(b) it is self-intersecting. The points $O_{i-1}, O_{i}$ lie on one of the two bisectors of the angle between $V_{i-1} V_{i}$ and $V_{i} V_{i+1}$ at $V_{i}$ (because both the circles $Q_{i-1}$ and $Q_{i}$ touch the lines $V_{i-1} V_{i}$ and $\left.V_{i} V_{i+1}\right)$. There are two such bisectors at $V_{i}$ and we may choose either. There is an internal bisector, which separates the points $V_{i-1}, V_{i+1}$, and an external bisector, which does not separate these two points. Define

$$
e_{i}= \begin{cases}1 & \text { if } O_{i} V_{i} O_{i-1} \text { is the internal bisector at } V_{i}, \\ -1 & \text { if } O_{i} V_{i} O_{i-1} \text { is the external bisector at } V_{i}\end{cases}
$$

Also define

$$
f_{i}= \begin{cases}1 & \text { if }\left[V_{i}, V_{i+1}\right] \text { is a locally convex edge of } P, \text { that is, } V_{i-1} \text { and } \\ & V_{i+2} \text { lie on the same side of the line } V_{i} V_{i+1}, \\ & \text { if }\left[V_{i}, V_{i+1}\right] \text { is an inflectional edge of } P, \text { that is, } V_{i-1} \text { and } \\ & V_{i+2} \text { lie on opposite sides of the line } V_{i} V_{i+1} .\end{cases}
$$

There are essentially six combinations of internal/external bisectors and locally convex/ inflectional edges. Three of these are shown in Fig. 14, and the reader will have no difficulty in drawing the other three cases. With angles $\theta_{i}$ and $\theta_{i+1}$ as marked, and $r_{i}>0$ as the radius of $Q_{i}$, in every case

$$
\frac{\left\|V_{i} U_{i}\right\|}{\left\|U_{i} V_{i+1}\right\|}=\frac{e_{i} e_{i+1} f_{i} r_{i} \cot \theta_{i}}{r_{i} \cot \theta_{i+1}}
$$

and so

$$
\prod \frac{\left\|V_{i} U_{i}\right\|}{\left\|U_{i} V_{i+1}\right\|}=\prod e_{i} e_{i+1} \prod f_{i} \prod \frac{\cot \theta_{i}}{\cot \theta_{i+1}} .
$$

Now $\prod e_{i} e_{i+1}=\prod e_{i}^{2}=1$ and $\prod f_{i}=1$ since the number of inflectional edges in a polygon must be even. This was proved by Fabricius-Bjerre [2]. In the last product all the terms cancel and so (4) is proved. 


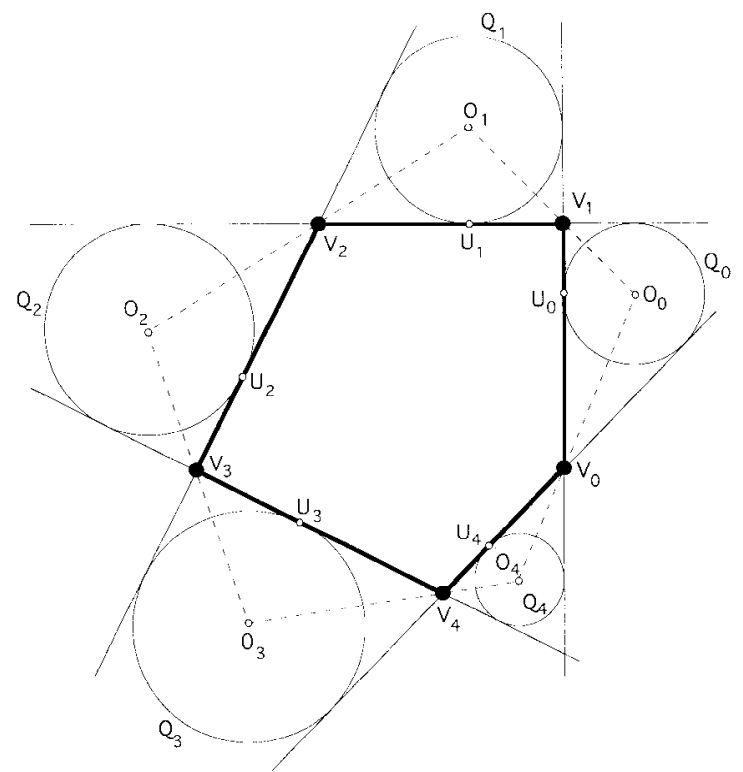

(a)

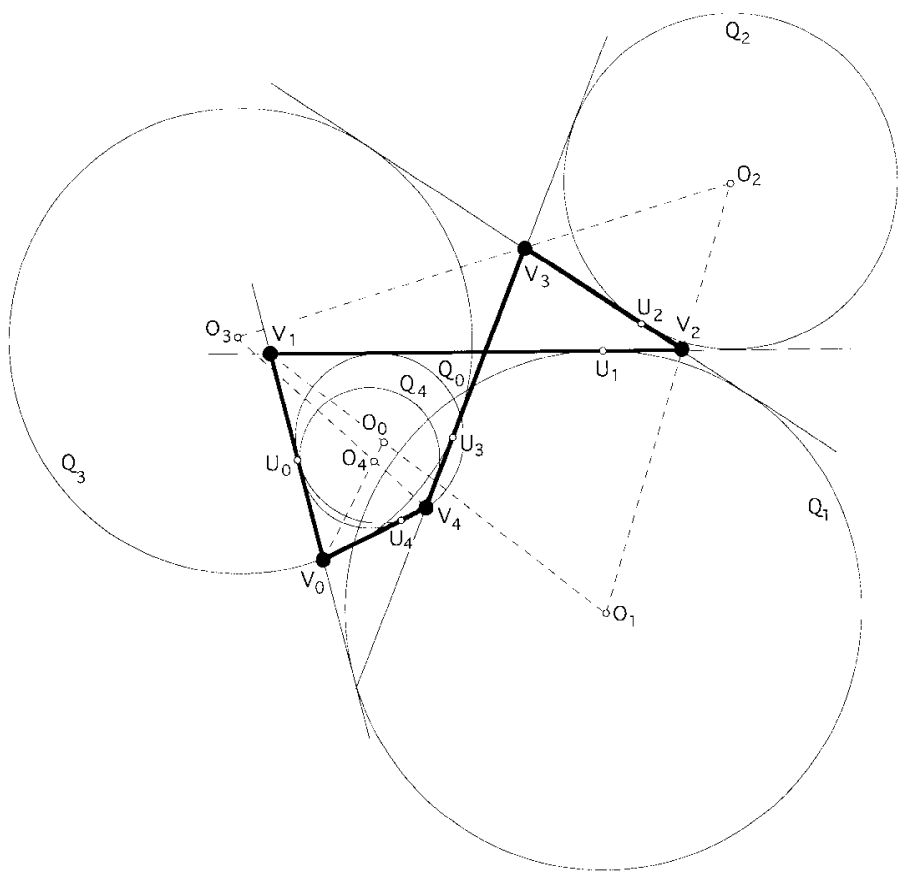

(b)

Fig. 13 


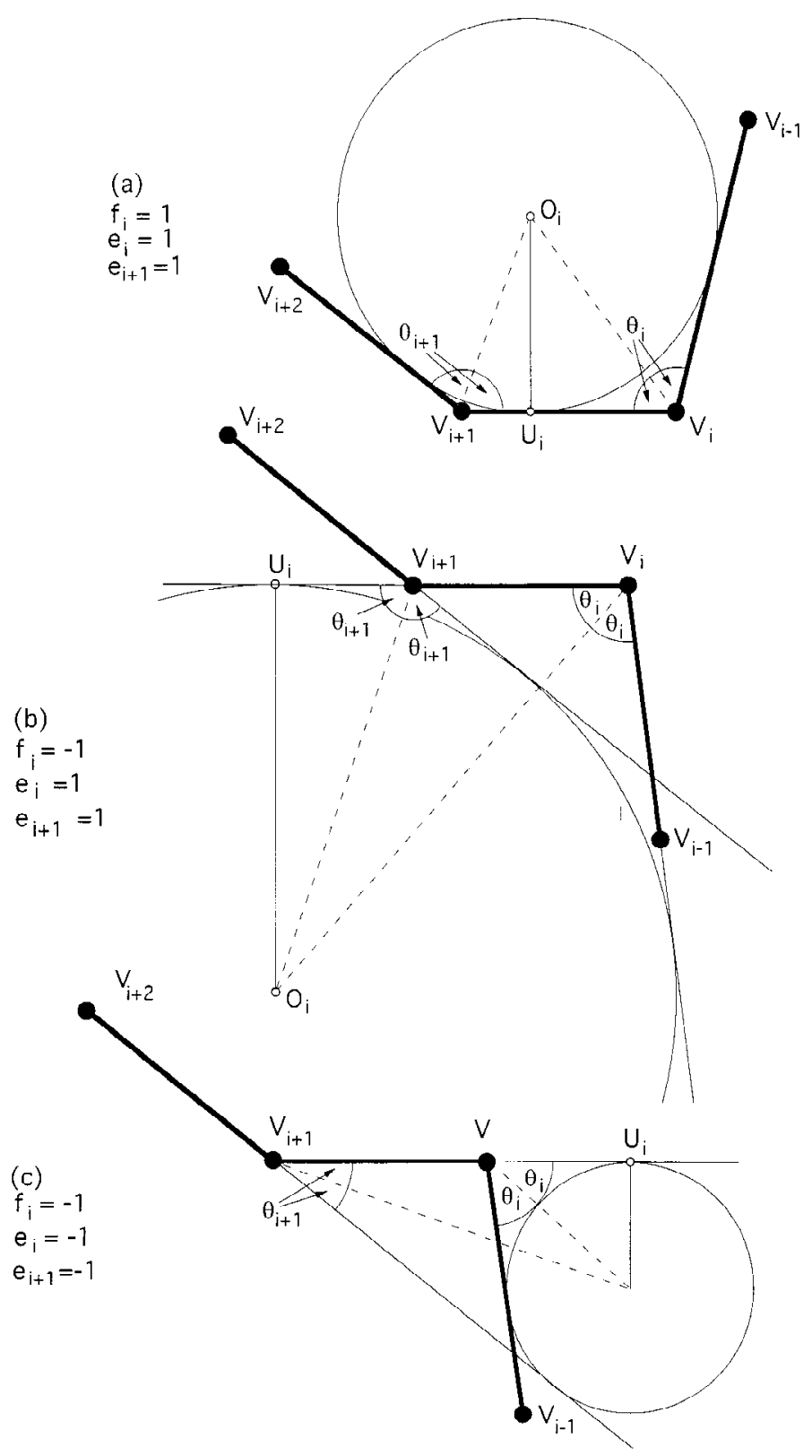

Fig. 14 
As in the previous theorem there are $2^{n}$ possible sets of bisectors, one at each of the vertices $V_{i}$. Each such set determines a sequence of circles $Q_{i}$ that satisfy the conditions of the theorem, since $O_{i}$, the centre of $Q_{i}$, is the point of intersection of the bisectors at $V_{i}$ and $V_{i+1}$. We thus arrive at $2^{n}$ sets of points $U_{i}$, each of which leads to a cyclic product relation. Hence, in general, (4) represents $2^{n}$ such relations.

Both Theorems 4.1 and 4.2 can be generalised in the following way. Instead of a sequence of $n$ circles $Q_{i}$ consider a sequence of $2 n$ circles $Q_{0}, \ldots, Q_{2 n-1}$ with the property that, for $i=0,1, \ldots, n-1$, in Theorem 4.1 the triples of points $O_{i}, F_{i+1}, O_{i+1}$ and $O_{i+n}, F_{i+1}, O_{i+n+1}$ (modulo $2 n$ ) are collinear, and in Theorem 4.2 the triples of points $O_{i}, V_{i+1}, O_{i+1}$ and $O_{i+n}, V_{i+1}, O_{i+n+1}$ (modulo $2 n$ ) are collinear. Defining the points $U_{i}(i=0, \ldots, 2 n-1)$ as before as the points of contact of these circles (with either $m$ or $V_{i} V_{i+1}$ ) (3) and (4) continue to hold except that our cyclic products now have $2 n$ terms. Similar generalisations to sequences of $3 n, 4 n, \ldots$, circles are clearly also possible.

Special cases of the above theorems occur when all the sides of $P$ touch a circle $Q$ (in Theorem 4.2) or all the sides of $P$ and the line $m$ touch a circle $Q$ (in Theorem 4.1). Then one possible sequence of circles $Q_{i}$ consists of the circle $Q$ repeated $n$ times. Condition (ii) becomes redundant, but the proofs still hold and both theorems are true in this case. If $n=3$ there are eight sequences of circles which satisfy the conditions of Theorem 4.2. Of these, four degenerate into a single circle (the incircle or three excircles of the triangle $P$ ). In this case the lines $V_{0} U_{1}, V_{1} U_{2}$ and $V_{2} U_{0}$ are concurrent and then $\prod\left(\left\|V_{i} U_{i}\right\| /\left\|U_{i} V_{i+1}\right\|\right)=1$ is a consequence of Ceva's theorem.

\section{Conclusion}

As stated in the Introduction, Branko Grünbaum proved and conjectured some of the results given here in his lecture notes [3]. To be precise, he proved Carnot's Theorem 1.1 using the idea of the power of a point with respect to a circle, as we did, and he conjectured Theorems 1.2-1.4. By the term "conjecture" we mean that he stated the results and then verified them numerically by computing the cyclic products for a large number of $n$-gons. He also proved Lemma 2 analytically (in contrast to the geometric method used here) and deduced Theorems 2.1 and 2.2 from it. All the other theorems in this paper are new.

All our theorems and proofs can be understood with a minimum of geometric knowledge. It is therefore surprising they were not discovered many years, or even centuries ago.

\section{Acknowledgement}

The author is indebted to the referees whose suggestions led to many improvements to this paper. 


\section{References}

1. Howard W. Eves, A Survey of Geometry, Allyn and Bacon, Boston, MA, 1972.

2. Fr. Fabricius-Bjerre, Proof of a relation between the numbers of singularities of a closed polygon, J. Geom., 13 (1979), 126-132.

3. Branko Grünbaum, unpublished lecture notes on "Modern Elementary Geometry" for a course given at the University of Washington, 1997.

4. Branko Grünbaum and G. C. Shephard, Ceva, Menelaus and the area principle, Math. Mat., 68 (1995), 254-268.

5. Branko Grünbaum and G. C. Shephard, Some new transversality properties, Geom. Dedicata, 71 (1998), 179-208.

6. R. A. Johnson, Advanced Euclidean Geometry (Modern Geometry), Houghton Mifflin, 1929. (Reprint: Dover, New York, 1960.)

Received December 1, 1998, and in revised form April 4, 1999. Online publication May 16, 2000. 